\title{
Thoracoscopic plication of the membranous portion of crescent-type tracheobronchomalacia in an elderly patient: a case report
}

Ryusuke Machino ${ }^{*}$ and Tsutomu Tagawa

\begin{abstract}
Background: It is presumed that tracheobronchomalacia in adults is caused by airway pressure-induced injury due to chronic cough related to pulmonary emphysema or chronic bronchitis. Commonly, a posterolateral approach using stabilizing materials is the surgical technique of choice for treating tracheobronchomalacia. We report a case in which thoracoscopic plication of the membranous portion was performed instead of airway stent placement for tracheobronchomalacia in an elderly individual.
\end{abstract}

Case presentation: An 87-year-old man who had been treated for bronchial asthma, pulmonary emphysema, and tracheobronchomalacia was admitted to our hospital with acute exacerbation of dyspnea. The patient underwent tracheal intubation, which was followed by tracheostomy 16 days later. Insertion of the tip of the adjustable-length tracheostomy tube to the end of the stenotic lesion enabled him to breathe spontaneously. However, conservative management failed due to recurrent pneumonia caused by the tracheobronchomalacia. Crescent-type tracheobronchomalacia (Johnson's classification grade III) was diagnosed, and the main narrowed area of the trachea was assumed to be approximately $3-10 \mathrm{~cm}$ from the tracheal bifurcation. A thoracoscopic approach was selected because a posterolateral approach was considered too invasive considering the patient's age and general condition. We placed eight stitches on the tracheal membranous portion and four stitches on the membranous portion of the right main bronchus, using the horizontal mattress suture technique. The use of foreign materials was avoided because meropenem-resistant Pseudomonas aeruginosa was cultured in a tracheal specimen. Immediately after the operation, the expiratory airway stenosis improved, and subsequently, spontaneous ventilation was possible using a normal type of tracheostomy tube instead of an adjustable-length tracheostomy tube.

Conclusions: Tracheobronchomalacia is not a rare condition in patients with chronic obstructive pulmonary disease. The thoracoscopic approach is less invasive than the posterolateral approach and is suitable in cases that are otherwise refractory to medical treatment. We believe that thoracoscopy may be a useful treatment option in cases where conservative treatment is not appropriate.

Keywords: Plication, Tracheobronchomalacia, Thoracoscopy

* Correspondence: groovemaker1981@yahoo.co.jp

Department of Thoracic Surgery, National Hospital Organization Nagasaki

Medical Center, 1001-1 Kubara, Omura, Nagasaki 856-8562, Japan

Springer Open
( ) The Author(s). 2020 Open Access This article is licensed under a Creative Commons Attribution 4.0 International License, which permits use, sharing, adaptation, distribution and reproduction in any medium or format, as long as you give appropriate credit to the original author(s) and the source, provide a link to the Creative Commons licence, and indicate if changes were made. The images or other third party material in this article are included in the article's Creative Commons licence, unless indicated otherwise in a credit line to the material. If material is not included in the article's Creative Commons licence and your intended use is not permitted by statutory regulation or exceeds the permitted use, you will need to obtain permission directly from the copyright holder. To view a copy of this licence, visit http://creativecommons.org/licenses/by/4.0/. 


\section{Background}

Tracheobronchomalacia in adults is believed to be caused by airway pressure-induced injuries due to chronic cough secondary to pulmonary emphysema or chronic bronchitis [1,2]. Masaoka et al. classified the disease into the following three types based on the etiology: pediatric, adult, and secondary types [3]. In terms of form, tracheobronchomalacia is classified as the saber-sheath type and the crescent-moon type (crescenttype). In the former type, the lateral diameter is shortened during exhalation, and both sidewalls are closed, whereas in the latter type, the transverse diameter expands during exhalation and the membranous portion is closed. The Johnson's classification divides airway lumen obstruction during coughing into the following four grades: grade I ( $50 \%$ or less), grade II (50-75\%), grade III (75-100\%), and grade IV (complete obstruction during coughing) [4]. Although conservative therapy is the mainstay of treatment for this disease, surgical treatment is considered in severe cases [5]. We report a case in which thoracoscopic plication of the membranous portion was performed for tracheobronchomalacia, instead of using foreign substances, in an elderly individual.

\section{Case presentation}

An 87-year-old man who had been treated for bronchial asthma, pulmonary emphysema, and tracheobronchomalacia (modified British Medical Research Council questionnaire grade III) at the department of internal medicine in our hospital was admitted for acute exacerbation of dyspnea. The patient's pulmonary function before hospitalization was as follows: vital capacity (VC), $1200 \mathrm{~mL}$; \% VC, 46\%; forced expiratory volume in one second (FEV1. 0), $500 \mathrm{~mL}$; and FEV1. 0\%, 45.7\%.

The patient underwent tracheal intubation, followed by tracheostomy 16 days later. Insertion of the tip of an adjustable-length tracheostomy tube to the end of the stenotic part enabled him to breathe spontaneously. However, it was difficult to continue conservative management because he repeatedly suffered from pneumonia with meropenem (MEPM)-resistant Pseudomonas aeruginosa. Sixty-five days after admission, he was referred to our department for surgical treatment.

The trachea was completely occluded during coughing. The crescent-type of tracheobronchomalacia (Johnson's classification grade III) was also diagnosed based on the findings of a bronchoscopic examination (Fig. 1a). Chest computed tomography (CT) revealed stenosis ranging from the trachea to the main bronchus (Fig. 1b). However, the patient could breathe spontaneously when an adjustable-length tracheal tube was inserted. Therefore, the main narrowed area of the airway was assumed to be approximately $3-10 \mathrm{~cm}$ from the tracheal bifurcation.
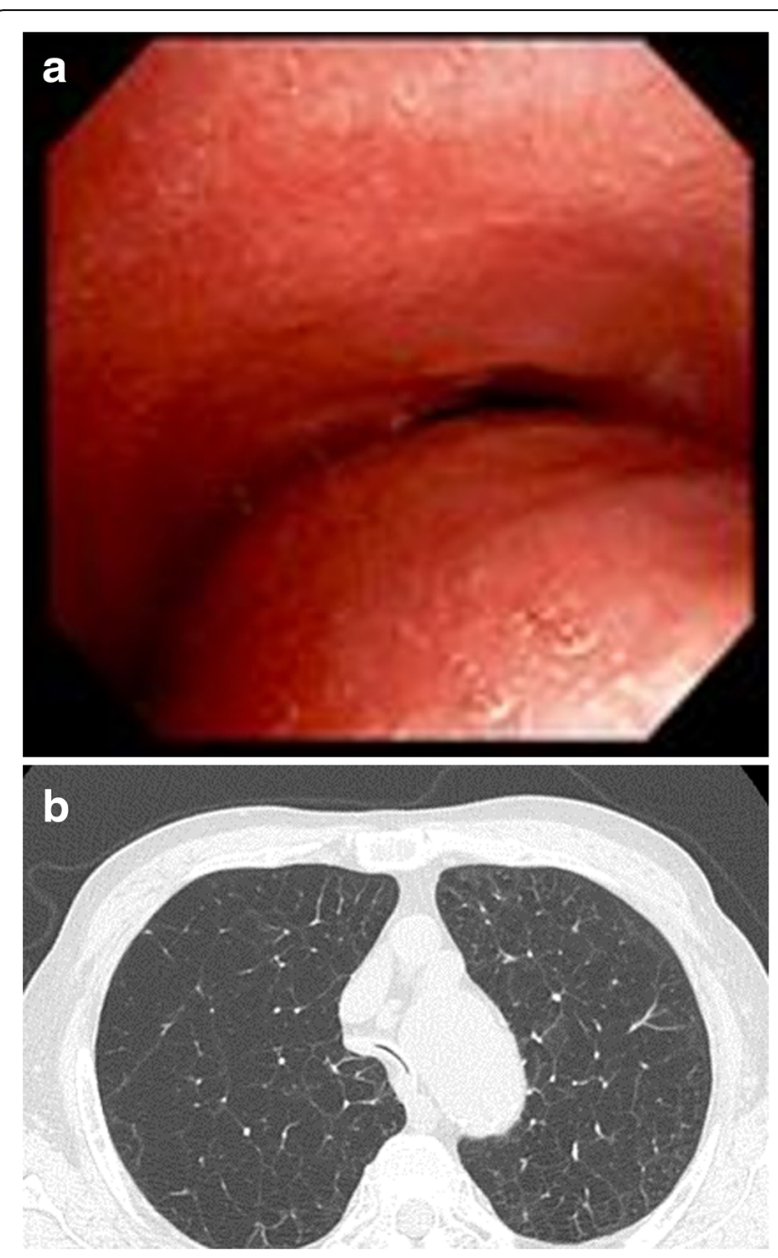

Fig. 1 Bronchoscopy and chest computed tomography (CT). a. Bronchoscopic examination showing occlusion during coughing. b Chest $\mathrm{CT}$ revealing stenosis ranging from the trachea to the right main bronchus

A posterolateral approach is commonly used for the plication or external fixation of the membranous portion in tracheobronchomalacia. In this case, we chose the thoracoscopic approach because the posterolateral approach was too invasive, considering the patient's age and general condition. He underwent thoracoscopic surgery with five ports (Fig. 2a). A satisfactory observation field was attained under differential lung ventilation with a double-lumen tube and carbon dioxide insufflation to an intrathoracic pressure of $5 \mathrm{mmHg}$. We exposed the trachea and the right main bronchus just below the right subclavian artery. Thereafter, we placed eight sutures on the tracheal membranous portion and four sutures on the right main bronchial membranous portion, using the horizontal mattress suture technique with 4-0 PDS double-ended needles (Fig. 2b, c). The trachea and the right main bronchus were plicated by pulling up the loosened membranous portion to avoid damage to the tracheal tube, and the size of the trachea was matched 

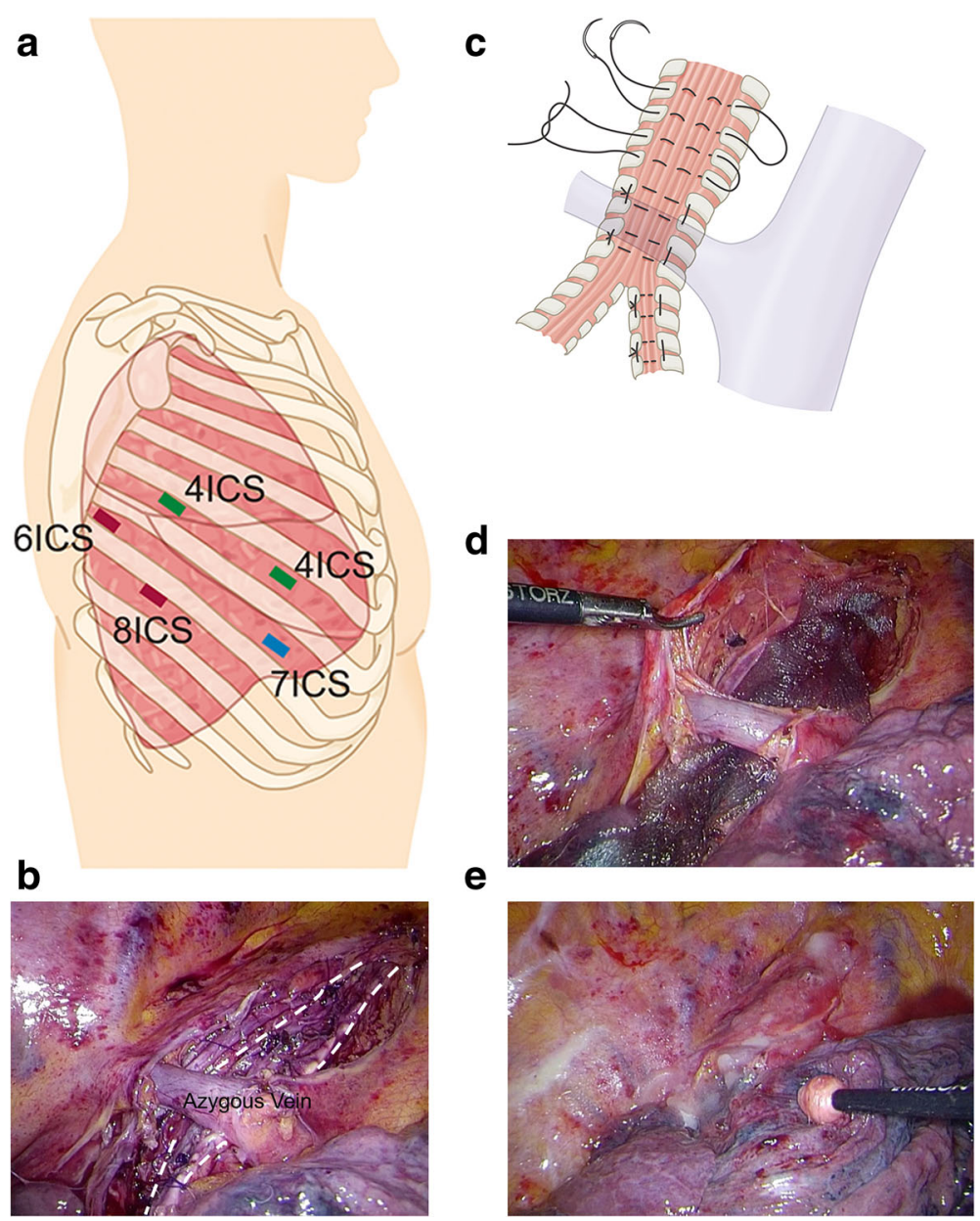

Fig. 2 Surgical technique. a Schema of port sites in this surgery. b Plicated tracheal and right main bronchial membranous portion using the horizontal mattress suture technique with 4-0 PDS double-ended needles (within dotted line). c Schema of the plication technique. d Polyglycolic acid sheets on the sutured portion, fixed with fibrin glue. e The plicated portion covered with the mediastinal pleura

with the size of the tracheal tube. The left main bronchus was not sutured. Additionally, we attached polyglycolic acid (PGA) sheets on the sutured portion using fibrin glue (Fig. 2d) and the mediastinal pleura (Fig. 2e) without suturing. We avoided the use of foreign materials as much as possible because MEPM-resistant Pseudomonas aeruginosa was cultured from a sample taken from the inside of the trachea. Immediately after the operation, the expiratory airway stenosis improved, and subsequently, spontaneous ventilation became possible with a normal type of tracheostomy tube instead of an adjustable-length tracheostomy tube. The severity of tracheomalacia improved to less than grade I in the Johnson's classification.

The patient needed long-term rehabilitation; however, he did not experience any difficulties in breathing, and the pneumonia was successfully treated. The patient was discharged on postoperative day 126 with a normal tracheostomy tube in situ. Bronchoscopy revealed that a preoperatively constricted area was present at 6 months after the operation (Fig. 3). The stenotic area was found to have expanded on chest CT. Despite avoiding the use of foreign materials, such as a mesh, the dorsal side of the membranous part was covered with a thickened scar tissue (Fig. 4a, b).

\section{Discussion}

We reported a case in which thoracoscopic plication of the membranous portion was performed instead of the use of foreign substances for tracheobronchomalacia in an elderly individual.

For tracheobronchial extracorporeal fixation, Nissen et al. described an operation using autologous ribs [6], and Herzog et al. described an operation using the rectus abdominal muscle sheath [7]. Subsequently, Wright et al. reported on extra-membranous external fixation using a polypropylene mesh [8. 9]. In this technique, in addition to direct fixation of the membranous portion 


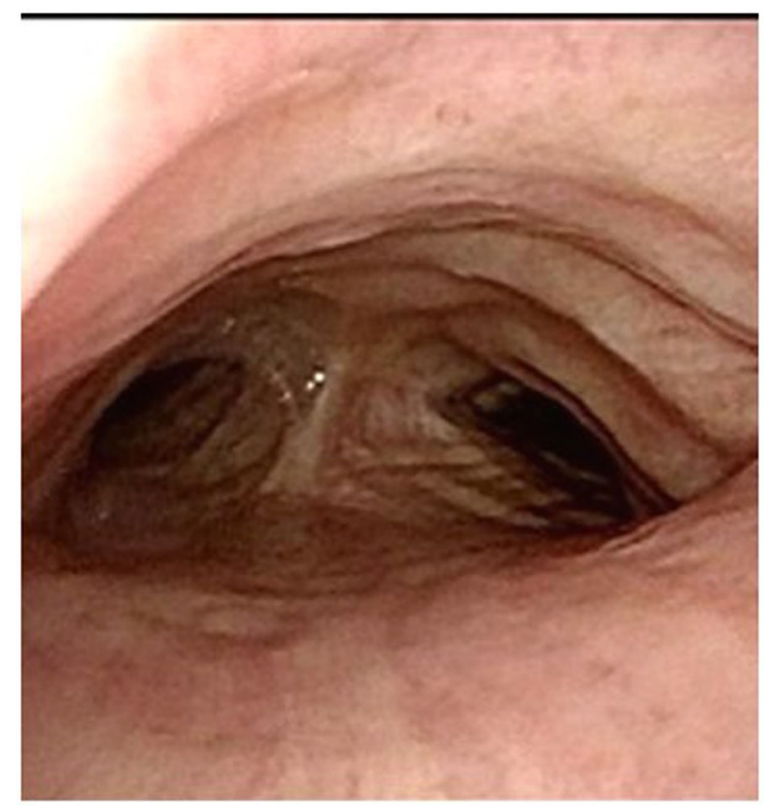

Fig. 3 Postoperative bronchoscopy. The area that had been constricted preoperatively remains constricted at 6 months postoperatively
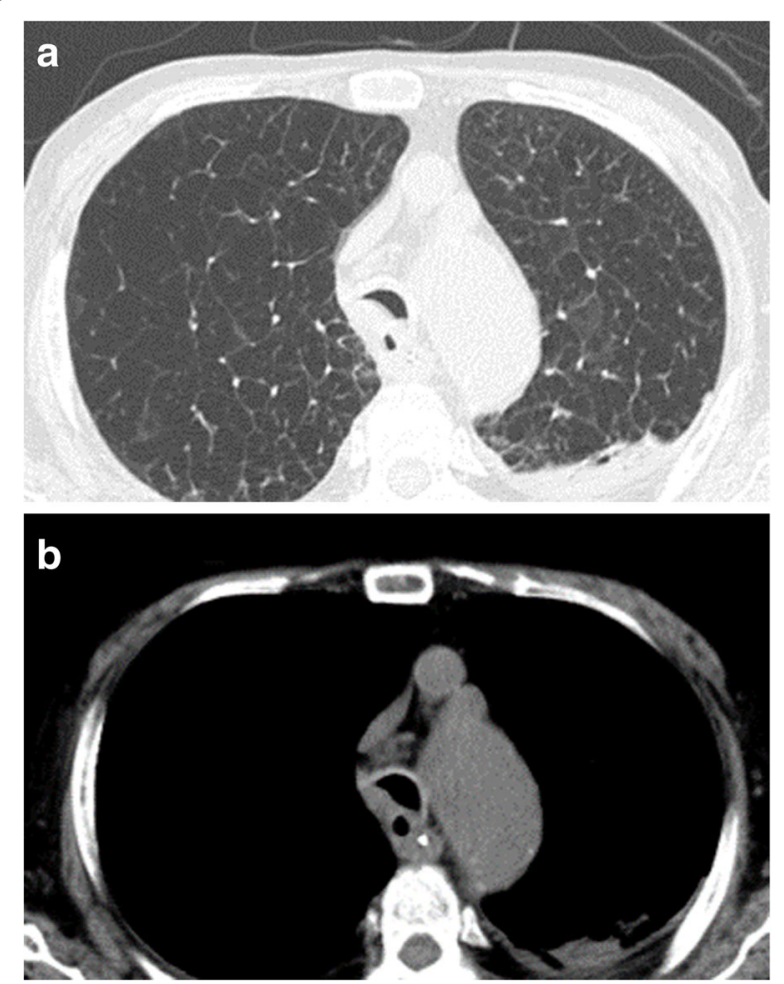

Fig. 4 Postoperative chest computed tomography (CT). a Expansion of the stenotic area on chest CT at 6 months postoperatively. b Scars covering the dorsal side of the membranous portion of the bronchial tube at 6 months postoperatively with a polypropylene mesh, the scar tissue grows, and the polypropylene mesh is integrated into the membranous portion to increase its stability and to maintain airway lumen patency [5]. Thus, fixation of the membranous portion with stabilizing materials, such as polypropylene meshes $[8,9]$ or polytetrafluoroethylene sheets [10], is the most common approach in recent years. In our case, after applying PGA sheets on the membranous portion, the area around the membranous portion was covered with thick scar tissue at 6 months after the operation although stabilizing materials were not used, and this also led to the stability of the membranous portion. The fixation was adequate, and restenosis was not observed. The suturing may cause restenosis long term; however, it is considered appropriate when performing lifesaving surgery in the elderly.

Indications for surgical treatment of tracheobronchomalacia include the absence of irreversible respiratory disorders, such as chronic obstructive pulmonary disease (COPD), and any other underlying disease that could complicate surgery. However, Ernst et al. reported the effectiveness of extra-membranous fusion using a polypropylene mesh for tracheobronchomalacia associated with severe emphysema. According to their report, airway stabilization is indicated in patients whose respiratory condition is improved by placing a silicone stent [11]. In the present case, assuming that the adjustablelength tracheostomy tube was equivalent to stent placement, it would have been considered an indication for surgery, because spontaneous breathing was possible by the placement of the adjustable-length tube.

In addition, this procedure is commonly performed using a conventional posterolateral approach. Most reports on this approach indicate that foreign materials such as polypropylene meshes $[8,9]$ are attached to the membranous portion. We aimed to reduce the invasiveness of the operation compared to that of the conventional posterolateral approach by performing it under thoracoscopy. The outcome of this less invasive, safer approach suggests that more patients can undergo this surgery. Additionally, we chose to avoid the use of foreign materials because MEPM-resistant Pseudomonas aeruginosa had been detected in the central airway. Hence, we decided to plicate the membranous portion with horizontal mattress sutures using absorbent threads at both ends of the needle and use only PGA sheets and fibrin glue for fixation. This method was adopted from the report by Masaoka et al. and modified [3]. We used horizontal mattress sutures in order to avoid tissue damage as much as possible and to increase the suture density to obtain a reliable forming effect. This method is advantageous because it avoids the use of foreign materials, and the procedure is simpler and easier to perform under thoracoscopy compared to establishing stabilization using a mesh. Although 
this method is considered useful for the crescent type of tracheobronchomalacia, it is necessary to fix the left and right cartilage parts for the saber-sheath type; hence, it is necessary to fix the trachea by a prosthesis.

First, we separated the lung ventilation by inserting an extra-long, single-lumen tube using an occlusion balloon, because the double-lumen tube was too large and could potentially be damaged by the suturing [9]. However, because the patient had COPD, the right lung was not sufficiently collapsed. Posterolateral thoracotomy allows for the surgery to be performed while isolating the lung that cannot be collapsed. In contrast, when performing thoracoscopic surgery, the single-lumen tube provides insufficient collapse of the lung. Thus, we provided unilateral lung ventilation with a double-lumen tube and carbon dioxide insufflation to create a sufficient operative field. Nevertheless, care must be taken to prevent damage to the tube-cuff during suturing. In addition, there have been several reports on azygous vein ligation and division $[9,12]$. However, using the thoracoscopic field of view, the posterior aspect of the azygous vein could be sutured without tension.

However, because the cervical trachea cannot be visualized using a transthoracic approach, a tracheostomy or a permanent stent may be required when treating patients in whom malacia or stenosis extends to the cervical trachea.

\section{Conclusions}

Plication of the membranous portion of crescent-type tracheobronchomalacia during thoracoscopic surgery results in similar outcomes compared to other approaches. The invasiveness of this procedure is much less than that of the posterolateral approach. We believe that thoracoscopy may be a useful treatment option in cases where conservative treatment is not appropriate.

\section{Abbreviations}

COPD: Chronic obstructive pulmonary disease; CT: Computed tomography; FEV1.0: Forced Expiratory Volume in one second; MEPM: Meropenem; PGA: Polyglycolic acid; VC: Vital capacity

\section{Acknowledgements}

We would like to thank Editage (http://www.editage.jp) for English language editing.

\section{Authors' contributions}

RM drafted the manuscript. RM and TT edited the article. RM and TT performed the preoperative investigation and operation. $T T$ provided the academic consideration. All authors read and approved the final manuscript.

\section{Funding}

No funding was used to support this study.

\section{Availability of data and materials}

The datasets supporting the conclusions of this article are included within the article.
Ethics approval and consent to participate

Ethics approval and consent to participate were not necessary due to the nature of the study.

\section{Consent for publication}

Written informed consent was obtained from the patient for publication of this report and for the use of the accompanying images.

\section{Competing interests}

The authors declare that they have no competing interests.

Received: 31 January 2020 Accepted: 31 March 2020

Published online: 06 April 2020

References

1. Nuutinen J. Acquired tracheobronchomalacia: a bronchological follow-up study. Ann Clin Res. 1977;9:359-64.

2. Murgu S, Colt H. Tracheobronchomalacia and excessive dynamic airway collapse. Clin Chest Med. 2013;34:527-55.

3. Masaoka A, Yamakawa Y, Niwa H, Hara F, Kondo S, Fukai I, et al. Pediatric and adults tracheobronchomalacia. Eur J Cardiothorac Surg. 1996;10:87-92.

4. Johnson TH, Mikita JJ, Wilson RJ, Feist JH. Acquired tracheomalacia. Radiology. 1973;109:576-80.

5. Grillo HC. Surgery for tracheomalacia, tracheopathia osteoplastica, tracheal compression, and staged reconstruction of the trachea. In: Grillo HC, editor. Surgery of the trachea and bronchi. Ontario: BC Decker; 2004. p. 645-63.

6. Nissen R. Tracheoplastik zur Beseitigung der Erschlaffungdes membraneosen Teils der intrathorakalen Luftrohre. Schweiz Med Wochenschr. 1954;84:219-21.

7. Herzog H, Keller R, Allgöwer M. Special methods of diagnosing and treating obstructive diseases of the central airways. Chest. 1971;60:49-67.

8. Wright CD, Grillo HC, Hammoud ZT, Wain JC, Gaissert HA, Zaydfudim V, et al. Tracheoplasty for expiratory collapse of central airways. Ann Thorac Surg. 2005;80:259-66.

9. Wright CD. Tracheobronchomalacia and expiratory collapse of central airways. Thorac Surg Clin. 2018;28:163-6.

10. Takazawa S, Uchida H, Kawashima H, Tanaka Y, Masuko T, Deie K, et al. External stabilization for severe tracheobronchomalacia using separated ring-reinforced ePTFE grafts is effective and safe on a long-term basis. Pediatr Surg Int. 2013;29:1165-9.

11. Ernst A, Odell DD, Michaud G, Majid A, Herth FFJ, Gangadharan SP. Central airway stabilization for tracheobronchomalacia improves quality of life in patients with COPD. Chest. 2011;140:1162-8.

12. Buitrago DH, Wilson JL, Parikh M, Majid A, Gangadharan SP. Current concepts in severe adult tracheobronchomalacia: evaluation and treatment. J Thorac Dis. 2017:9:E57-66.

\section{Publisher's Note}

Springer Nature remains neutral with regard to jurisdictional claims in published maps and institutional affiliations.

\section{Submit your manuscript to a SpringerOpen ${ }^{\circ}$ journal and benefit from:}

- Convenient online submission

- Rigorous peer review

- Open access: articles freely available online

- High visibility within the field

- Retaining the copyright to your article

Submit your next manuscript at $\boldsymbol{\nabla}$ springeropen.com 